\title{
CONCEPTUALIZACIÓN DEL COMPORTAMIENTO EMOCIONAL Y LA ADICCIÓN A LAS REDES SOCIALES
}

\section{CONCEPTUALIZATION OF EMOTIONAL BEHAVIOR AND ADDICTION TO SOCIAL NETWORKS}

Jose A. García del Castillo , Álvaro García del Castillo-López¹, Paulo C. Dias² y Fernando García-Castillo³

\author{
${ }^{1}$ Universidad Miguel Hernández, España \\ ${ }^{2}$ Universidade Católica de Braga, Portugal \\ ${ }^{3}$ Universidad de Alicante, España
}

\section{Abstract}

In a few years, social networks have become an instrument of influence and communication in all age ranges. Preteens and adolescents remain the most vulnerable group because they lack strategies to defend themselves against certain aggressions of this environment. The information and training channels for the management of social networks are still scarce and controversial and their power of social influence is evident in different areas of society, generating currents of opinion and modification of attitudes and beliefs. The great versatility and ease of use, makes them instruments for continued use. We have to add to the process the ignorance on the part of the parents of how to educate the children in their use, taking into account that there is a quite pronounced digital divide. The emotional states in this age group are in continuous development and any incident can have a positive or negative impact on their configuration and, on the other hand, the consequences of an abusive use of them can lead to addiction behavior. In this work we intend to address, from the review of the literature and from a theoretical point of view, the influence of virtual social networks on the emotional behavior of young people and their possible relationship with the addiction process.

Keywords: Social Networks, Adolescents, Emotional Behavior, Addiction Process.

\section{Resumen}

Las redes sociales se han convertido en muy pocos años en un instrumento de influencia y comunicación en todos los rangos de edad. Los preadolescentes y adolescentes siguen siendo el grupo más vulnerable porque carecen de estrategias para defenderse de determinadas agresiones de este medio. Los canales de información y formación para el manejo de las redes sociales siguen siendo aún escasas y controvertidas y su poder de influencia social se evidencia en diferentes ámbitos de la sociedad, generando corrientes de opinión y modificación de actitudes y creencias. La gran versatilidad y facilidad de utilización, las convierte en instrumentos de uso continuado. Tenemos que añadir al proceso el desconocimiento por parte de los padres de cómo educar a los hijos en su uso, teniendo en cuenta que existe una brecha digital bastante pronunciada. Los estados emocionales en este grupo de edad se encuentran en continuo desarrollo y cualquier incidente puede repercutir positiva o negativamente en su configuración y, por otra parte, las consecuencias de un uso abusivo de las mismas, puede conllevar una conducta de adicción. En este trabajo pretendemos abordar, a partir de la revisión de la literatura y desde un punto de vista teórico, la influencia de las redes sociales virtuales en el comportamiento emocional de los jóvenes y su posible relación con el proceso de adicción.

Palabras clave: Redes Sociales, Adolescentes, Comportamiento Emocional, Proceso de Adicción.

Correspondence: Jose A. García del Castillo | jagr@umh.es 
En la actualidad no entenderíamos el mundo de las relaciones interpersonales sin las redes sociales virtuales. Su poder de comunicación e influencia es un hecho incuestionable en cualquier rango de edad, sobre todo, por la facilidad de uso y la accesibilidad. Según Serrano (2013), a pesar de la gran diferencia potencial entre las relaciones sociales reales y virtuales, contamos con alguna evidencia científica que considera ambas relaciones en el mismo nivel de importancia.

Los espacios virtuales han generado una nueva forma de relación donde no es necesaria la presencia física y donde se posibilita la ausencia de la identidad de los usuarios, lo que conlleva una libertad total de expresión. Los procesos de influencia entre usuarios son altos y no siempre recíprocos. Como señalan González y Martínez (2018), también funcionan como filtros y alertas, permitiendo a los usuarios modular la información en función de sus intereses y seleccionando aquellos usuarios que les inspiran más confianza y/o influencia.

Las redes sociales virtuales han transformado y relativizado los conceptos de tiempo y espacio, sobre todo para la población más joven. Como señala Ferreiro (2015), el cambio en la percepción del tiempo se materializa en la inmediatez. Santos (2018) desarrolla la idea en base a la densidad. En la actualidad se percibe el tiempo y el espacio como algo cercano y rápido, frente a lo que sucedía antaño donde los tiempos estaban mediatizados por las distancias y eran mucho más densos.

La influencia de las redes sociales virtuales abarca grandes áreas del desarrollo modificando y/o alterando el comportamiento real de los jóvenes. Podemos observar procesos de influencia en referencia a la construcción de la identidad (Cantor, Pérez \& Carrillo, 2018; González \& Martínez, 2018; Khan, Gagné, Yang, \& Shapka, 2016; Pérez, Pastor, \& Abarrou, 2018), a las conductas de sueño (Li, Kawachi, Buxton, Haneuse \& Onnela, 2019; Li \& Lu, 2015; Puerto, Rivero, Sansores, Gamboa, \& Sarabia, 2015); al rendimiento escolar (Alwagait, Shahzad, \& Alim, 2015; Badri, Nuaimi, Guang, \& Rashedi, 2017; Lizárraga, Rodelo, Tovar, \& Aguiano, 2015); al consumo de drogas (Golpe, Gómez, Braña, Varela, \& Rial, 2017; Martinez, Facundo, Cardenas, Castillo, \& Garcia, 2017; Navarro, De Anda, Gámez, Candía, \& Guzmán, 2016; Nesi, Rothenberg, Hussong, \& Jackson, 2017; Sampasa \& Hamilton, 2018); a las emociones (Li,
Chang, Chua, \& Loh, 2018; Radovic, Gmelin, Stein, \& Miller, 2017; Throuvala, Griffiths, Rennoldson, \& Kuss, 2019); al comportamiento sexual (Cookingham \& Ryan, 2015; McCann, Broccatelli, Moore, \& Mitchell, 2018; Peris, Maganto, \& Kortabarria, 2013); a la adicción a las redes sociales (Banyai, Zsila, Kiraly, Maraz, Elekes, Griffiths, Andreassen, \& Demetrovics, 2017; Rojas, Ramos, Pardo, \& Henríquez, 2018; Soh, Chew, Koay, \& Ang, 2018; Tang \& Koh, 2017); a la conducta alimentaria (Holland \& Tiggemann, 2016; Mingoia Hutchinson, Wilson, \& Gleaves, 2017; Restrepo \& Castañeda, 2018; Turner \& Lefevre, 2017); a la salud (Carrotte, Vella, \& Lim, 2015; Park, Sun, \& McLaughlin, 2017; Raudsepp \& Kais, 2019; Sampasa \& Hamilton, 2015); entre otros comportamientos y procesos.

Nuestro objetivo en este trabajo es abordar a partir de la revisión de la literatura y desde un punto de vista teórico, la influencia de las redes sociales virtuales en el comportamiento emocional de los jóvenes y su posible relación con el proceso de adicción.

\section{Influencia de las redes sociales virtuales en los jóvenes}

El embrión de las redes sociales virtuales, se planteó desde las ciencias sociales con mucha antelación a la irrupción de las nuevas tecnologías de la comunicación y la información (TIC). Los antecedentes de las redes sociales en Internet se pueden secuenciar en tres momentos:

- El cuento de Frigyes Karinthy (1929), donde desarrolla la idea de que una persona a partir de un reducido número de contactos puede crear una cadena exponencial que una a toda la humanidad.

- El experimento de Milgram (1967) para probar la teoría que denominó “el problema del mundo pequeño". Su conclusión fue que serían necesarios seis pasos de promedio para conectarse con cualquier persona de los EEUU.

- Finalmente, Watts (2003) basándose en el experimento de Milgram desarrolla la teoría de los seis grados de separación. Teóricamente se necesitan una media de seis intermediarios en la relación entre dos personas de cualquier parte del mundo. 
En la actualidad podemos afirmar que las redes sociales han conseguido convertirse en muy pocos años en referencia de comunicación entre los jóvenes. Como afirman Sanz et al. (2018), es la actividad preferente de los jóvenes españoles en sus momentos de ocio digital. Según estos autores dos de cada tres jóvenes de secundaria, publica frecuentemente en las redes sociales sobre: fiestas y celebraciones (24\%), prácticas físicas y deportivas (20\%) y actividades culturales (18\%). Aunque parece que las redes sociales han dejado de ser únicamente una opción de diversión destinada al tiempo libre para convertirse en una obligación, una necesidad. No participar en las redes sociales equivale a no tener presencia en la sociedad (Díaz, 2011; Salas \& Escurra, 2014).

Por otra parte, las redes sociales ejercen una influencia tan potente en la población joven, que es muy difícil supervisar, por parte de los padres y educadores, todos sus movimientos. De hecho, es cada vez más usual que los menores utilicen dispositivos móviles con acceso a Internet fuera del control de los padres. La repercusión que hoy en día tiene la brecha digital en la autoridad está definida primordialmente por la ausencia de conocimientos y habilidades de los padres para poder ejercer dicha autoridad de un modo comprometido y responsable (López-Sánchez \& García del Castillo, 2017).

Las redes sociales han cambiado sustancialmente la forma de percibir y vivir la realidad social y la comunicación interpersonal. Como afirma Carr (2013), la interactividad de la red ha conseguido que se convierta en el centro neurálgico de todas las reuniones del mundo, donde se juntan para charlar, presumir, conocer gente a través de todo tipo de redes sociales (a veces más antisociales que sociales). La comunicación cara a cara ha pasado a un segundo plano, según indican Salas y Escurra (2014):

- Los jóvenes "hablan" a través de mensajes.

- "Conversan" mediante la lectura y la escritura.

- Se "encuentran" con amigos y familiares a distancia.

Queda patente, que las redes sociales se han convertido en una experiencia normativa que puede influir significativamente en el desarrollo psicológico y social de los jóvenes (Pertegal, Oliva, \& Rodríguez, 2019).
Según estos autores los jóvenes españoles tienen una actitud positiva hacia el uso de redes sociales y miedo a perderse algo si no están conectados. En general los motivos de uso giran en torno a:

- Necesidad de mantenerse en contacto.

- Establecer nuevas relaciones.

- Búsqueda incesante de reconocimiento social.

En esta misma línea, Hawk, van den Eijnden, van Lissa \& ter Bogt (2019), afirman que las tendencias narcisistas de los jóvenes son predictoras de un uso de las redes sociales para obtener atención y reconocimiento.

Las redes sociales desde el punto de vista de Gagner \& Davis (2014), se enmarcan en el mundo adolescente desde lo que los autores Ilaman los "nativos digitales", nacidos y educados en la era de las tecnologías. Desarrollan tres elementos fundamentales en su estudio, lo que denominan las tres íes:

- Identidad, entendida como algo prefabricado y deformado, ya que los usuarios intentan transmitir una imagen positiva de sí mismos.

- Intimidad, como una dimensión que posibilita el establecimiento de relaciones en cantidad y variedad, pero sin garantías en la calidad.

- Imaginación, referida a la forma en que los jóvenes emplean sus capacidades cognitivas, sociales y emocionales de una forma poco convencional.

\section{Comportamiento emocional de los jóvenes en las redes sociales virtuales}

Los jóvenes son un grupo de edad vulnerable a las influencias del entorno en el que se desarrollan. Su tasa de vulnerabilidad está en función de diferentes parámetros, como su integración social, su grado de educación, su entorno familiar entre otros. Por otra parte, la vulnerabilidad de los adolescentes está íntimamente unida a la percepción de riesgo.

Se asume que los adolescentes tienen una percepción de riesgo baja o muy baja a las situaciones de peligro y asumen con tranquilidad esos riesgos presuponiendo que son invulnerables a los mismos. 
Como indicábamos en otros trabajos (García del Castillo, 2015), la vulnerabilidad que una persona puede acumular en función del riesgo, es directamente proporcional a la cantidad de exposición al mismo.

Partiendo de esta premisa, si los jóvenes pasan una media de 6 horas utilizando el smartphone (el $47 \%$ de ellos en redes sociales) (Dias et al., 2019), la probabilidad de que aumente su índice de vulnerabilidad a los riesgos asociados al uso es muy alto.

En esta línea, el trabajo de Livingstone \& Smith (2014), señala que:

- Más del 30\% de los menores de 18 años no tiene información para navegar seguro en Internet.

- Más del 30\% ha visitado páginas pornográficas de manera accidental.

- El $25 \%$ ha recibido pornografía vía correo electrónico.

- Entre un 40-70\% ha suministrado información personal vía web.

- El $40 \%$ de los menores de 18 años ha mentido sobre su edad para chatear o acceder a una cuenta de red social.

Las medidas de protección ante esta vulnerabilidad tienen que proceder de los padres y adultos de referencia. Pero como hemos comentado anteriormente existe una brecha digital importante entre generaciones que impide el desarrollo de una línea educativa eficaz.

Según Gros (2005), no hay que educar a los niños limitando el acceso a los medios digitales sino enseñar su utilización y conseguir que sean críticos y reflexivos. Intentar proteger a los niños y adolescentes limitándoles el acceso a Internet y las redes sociales es algo que está abocado al fracaso.

Estudios sobre la brecha digital generacional sostienen que son los adolescentes los que enseñan y educan a sus padres, lo que genera una alteración en las jerarquías familiares y deja a los menores en riesgo de uso y exposición, ya que los padres tienen pocas estrategias para una adecuada supervisión y control (Arab \& Díaz, 2015; López-Sánchez \& García del Castillo, 2017; Moreno, González, Torres, \& Araya, 2017).
Cuando aumenta el uso de redes sociales se "viralizan" determinadas emociones por parte de los usuarios por encima de otros contenidos. Berger \& Milkman (2012) sostienen en su investigación que la carga viral es mucho mayor cuando es emocional y, sobre todo, las que tienen componentes emocionales positivos (Tabla $1)$.

Tabla 1. "Viralización" de las emociones en las redes sociales virtuales

\begin{tabular}{lcc}
\hline Emoción & Positiva & Negativa \\
\hline Alta agitación & $\begin{array}{c}\text { Sorpresa } \\
\text { Entusiasmo }\end{array}$ & $\begin{array}{c}\text { Enfado } \\
\text { Ansiedad }\end{array}$ \\
\hline Baja agitación & Alegría & Tristeza
\end{tabular}

Alegría Tristeza

"Viralidad"

Fuente: Elaboración propia

De hecho, los estados emocionales positivos y negativos se transfieren a otros mediante el contagio emocional. Paradójicamente la exposición a la felicidad de los demás, puede ser deprimente, produciendo un efecto de comparación social (Kramer, Guillory, \& Hancock, 2014).

La percepción de emociones en las redes sociales virtuales tiende a interpretarse por los receptores como emociones negativas. En un estudio de Lucio (2012) con una amplia muestra de estudiantes, comprobó que más del $81 \%$ percibía en los demás, emociones de tristeza, depresión y soledad. Pero lo más relevante es que para más del 34\% de los participantes recibir emociones negativas a través de las redes sociales les provoca estados depresivos, tristeza e incertidumbre.

En un estudio reciente (De la Villa \& Suarez, 2016), el uso frecuente del teléfono móvil se configura como una de las tecnologías más desadaptativas, provocando en los adolescentes desajustes de autorregulación en las emociones negativas, déficits en las habilidades para afrontar situaciones estresantes y problemas de regulación emocional en general. 
En esta misma línea, se observa que el uso excesivo y la falta de autocontrol de las redes sociales virtuales incide en la generación de relaciones sociales problemáticas, así como problemas emocionales, de salud en general y de rendimiento académico y/o laboral. Por otra parte, puede convertirse en un impulsor determinante en la generación de depresión en los usuarios, con alteración en la autopercepción, comportamientos narcisistas y déficits en las relaciones sociales (Buran \& Dogan, 2019).

Así mismo el uso abusivo de las redes sociales virtuales tiende a generar problemas de baja autoestima, desconfianza en los demás, cambios bruscos y repentinos de humor, angustia psicológica, ideación suicida e intento de suicidio en los casos más extremos cuando aparecen problemas de acoso (Arab \& Dias, 2015; Bennett \& O'Donohue, 2014; Sampasa \& Chaput, 2016; Singleton, Abeles, \& Smith, 2016).

Algunos estudios sugieren que el aumento del estrés con el uso de las redes sociales virtuales depende en gran medida del miedo que se genera en el usuario a no ser reconocido suficientemente en las redes y carecer de una popularidad que se ajuste a sus expectativas de éxito social (Beyens, Frison, \& Eggermont, 2016).

Otras respuestas emocionales que se desprenden del uso y/o abuso de las redes sociales virtuales según Alonso \& Romero (2017), son las que marcan conductas anómicas y agresivas, un grado de impulsividad elevado, comportamientos poco amables y cerrados, dificultades en las relaciones interpersonales e inestabilidad emocional manifiesta.

\section{Adicción a las redes sociales virtuales}

La adicción a Internet y a las redes sociales virtuales, no aparecen incluidas en los criterios de adicción del DSM $\checkmark$ por falta de evidencias científicas, aunque muchos investigadores, cada vez más numerosos, se inclinan por considerarlas una adicción dado que cuentan con muchos de los indicadores prototípicos de otras adicciones (generación de conflictos, dilatado tiempo de uso, recaídas, etc.) en la misma línea de otras adicciones comportamentales.

La potencial adicción a las redes sociales virtuales se desprende de las amplias posibilidades de uso, su funcionalidad, su gran atractivo para las relaciones interpersonales, la gran facilidad de conexión y los refuerzos inmediatos que se obtienen (García del Castillo, 2013).

Un primer paso para determinar una potencial adicción a las redes sociales virtuales sería el de diferenciar entre hábito y adicción. Los usuarios de las redes sociales entienden que su utilización impulsa significativamente la socialización con otros usuarios. Este hecho hace que el uso se convierta en algo automático y consciente para ellos, es decir, en algo habitual. Los investigadores consideran que los usuarios de redes sociales confrontan los pros y los contras de su uso para conseguir sus metas de socialización, pero desde un plano de hábito. El problema puede surgir cuando se pasa de lo habitual automático a la adicción. Desde la teoría de la sensibilización de incentivos se podría dar explicación al paso del hábito a la adicción, dado que se pasa de un comportamiento inicialmente controlado a otro completamente compulsivo, asociado con las consecuencias altamente gratificantes de este comportamiento (Seo \& Ray, 2019).

Uno de los problemas fundamentales a la hora de llegar a una delimitación del concepto de adicción en las redes sociales virtuales, es la gran cantidad de terminología que se utiliza por parte de los investigadores para concretar el problema. En una revisión de Frost y Rickwood (2017), encontraron multitud de términos para referirse al uso patológico de las redes sociales virtuales, desde uso problemático a uso compulsivo, pasando por el propio término de adictivo. Además, los marcos teóricos desde los que parte son numerosos, por lo que es improbable que podamos llegar a una conclusión colegiada de este problema.

Apoyando la tesis de una posible adicción a las redes sociales virtuales encontramos diferentes estudios donde los altos índices de neuroticismo e impulsividad en el uso excesivo de redes sociales tiene una relación alta con conductas adictivas (Malo, Martín, \& Viñas, 2018; Marino, Vieno, Pastore, Albery, Frings, \& Spada, 2016; Tang, Chen, Yang, Chung, \& Lee, 2016).

Otros autores establecen una relación entre el riesgo de adicción a las redes sociales y la autoestima y el autocontrol. Cuanto menor es la autoestima y el autocontrol, mayor será el riesgo de adicción a las redes sociales y viceversa. Los jóvenes inseguros, que se encuentra más aislados de su círculo social y que tienen 
poco dominio sobre su comportamiento cuentan con un mayor riesgo de adicción a las redes sociales (Challco, Rodríguez, \& Jaimes, 2016). Así mismo encontramos correlaciones positivas entre dependencia emocional, abuso de Internet y móvil, ansiedad y depresión. Según los autores este perfil coincide con adolescentes que abusan de sustancias (Estévez, Urbiola, Iruarriazaga, Onaindia, \& Jauregui, 2017).

Según un estudio reciente de Buran \& Dogan (2019), aparecen diferencias de género en cuanto a la posible adicción a las redes sociales virtuales, siendo las mujeres más propensas que los hombres, sobre todo, buscando mejorar su estado de ánimo, así como estar más ocupadas en tareas de relaciones sociales. Comprueban que la adicción a las redes sociales es directamente proporcional al número de seguidores de Instagram. Finalmente observan una relación negativa entre los niveles de autoestima y la adicción a las redes sociales.

En una investigación con una muestra muy amplia de adolescentes (Chou \& Chou, 2019), los autores afirman que existe una clara diferenciación entre los usuarios que utilizan Internet para fines académicos frente a los que lo hacen para relaciones en redes sociales y otros fines recreativos. Según los autores, los que persiguen fines académicos no tienen una probabilidad muy baja de llegar a ser adictos y los que lo hacen con fines recreativos y de relación interpersonal su probabilidad es muy alta. También señalan que el factor que más probabilidad tiene de generar adicción son las compras en línea. Así mismo los adolescentes que acceden a las redes sociales virtuales tienen una probabilidad mayor de llegar a la adicción. Otros autores como López-Fernández (2014) corroboran estos resultados, pero también encontramos autores como Bae (2017) que entra en contradicción con los estudios anteriores.

Según un estudio reciente realizado con el MULTICAGE en población adulta (Pedrero, Ruiz, Rojo, Llanero, Pedrero, Morales, \& Puerta, 2018), los índices de dependencia que sugieren los autores son de más del $8 \%$ de adicción a Internet, casi un 11\% de adicción a la mensajería instantánea y más de un $6 \%$ de adicción a las redes sociales. Los autores postulan la indefinición en el abuso de las TIC y, dentro de ellas las redes sociales, como un problema de adicción.

No todos los autores están en la línea de que el abuso de las redes sociales virtuales acabe en una adicción. Carbonell \& Oberst (2015), afirman que efectivamente los jóvenes pueden usar en exceso las redes sociales en línea, pero no llegar nunca a la adicción.

Asumir con el término adicción cuando nos referimos al uso abusivo a Internet y/o las redes sociales en línea, puede convertirse en una construcción inadecuada por el hecho de etiquetar, más que por que se tenga certeza de que realmente estamos hablando de una adicción. Algunos autores (Carbonell, Chamarro, Oberst, Rodrigo, \& Prades, 2018) siguen señalando que sería más apropiado continuar hablando de abuso más que de adicción. Estos autores inciden en que el uso problemático no depende de las nuevas tecnologías en sí mismas sino de las acciones que se realizan con ellas. Así mismo indican que al etiquetar con el término adicción se puede llevar al campo de lo patológico en la vida cotidiana.

\section{A modo de conclusión}

Contamos con suficientes evidencias científicas de que las redes sociales virtuales se han convertido en una "necesidad" en el comportamiento habitual de los adolescentes y jóvenes. También se verifica que intervienen en procesos básicos de desarrollo, de influencia y de salud que pueden derivar en diferentes problemáticas.

Ante la demostrada vulnerabilidad de los jóvenes y adolescentes a los riesgos de las redes sociales, se aboga por la enseñanza en lugar de la limitación. Potencialmente parece que las redes sociales se pueden asociar a diferentes comportamientos emocionales: Desajustes, estrés, depresión, autoestima, desconfianza, ideas de suicidio, miedo, entre muchos otros.

Por otra parte, la controversia, en cuanto a la posible adicción de las redes sociales virtuales, sigue siendo un hecho, dado que no contamos con suficiente evidencia científica para hablar de adicción a las redes sociales virtuales, pero tampoco para dejar de hacerlo, teniendo en cuenta que muchos estudios apuntan esta posibilidad y la catalogan como tal. Creemos que sería conveniente ajustar la terminología y llegar a un acuerdo consensuado de qué entendemos por adicción a las redes sociales virtuales y diferenciar más claramente entre un uso, un abuso y una adicción a las mismas. 
Se demuestra ampliamente en la literatura científica que las redes sociales virtuales cuentan con una amplia influencia en el comportamiento emocional de los adolescentes y son potencialmente adictivas, por lo que sería conveniente poner en marcha las herramientas preventivas necesarias para reducir el impacto negativo y potenciar al máximo el positivo.

\section{REFERENCES}

Alonso, C. \& Romero, E. (2017). El uso problemático de nuevas tecnologías en una muestra clínica de niños y adolescentes. Personalidad y problemas de conducta asociados. Actas Españolas de Psiquiatria, 45(2), 62-70.

Alwagait, E., Shahzad, B., \& Alim, S. (2015). Impact of social media usage on students academic performance in Saudi Arabia. Computers in Human Behavior, 51(B), 1092-1097.

Arab, E. \& Díaz, A. (2015). Impacto de las redes sociales e Internet en la adolescencia: Aspectos positivos y negativos. Revista de Medicina Clínica Condes, 26(1), 7-13.

Badri, M., Nuaimi, A., Guang, Y., \& Rashedi, A. (2017). School performance, social networking effects, and learning of school children: Evidence of reciprocal relationships in Abu Dhabi. Telematics and Informatics, 34(8), 1433-1444.

Bae, S.M. (2017). The relationship between the type of smartphone use and smartphone dependence of Korean adolescents: National survey study. Children and Youth Services Review, 81,207-211. doi:10.1016/j.childyouth.2017.08.012

Banyai, F., Zsila, A.', Kiraly, O., Maraz, A., Elekes, Z., Griffiths, M.D., Andreassen, C., \& Demetrovics, Z. (2017). Problematic Social Media Use: Resultsfroma Large-Scale Nationally Representative. Adolescent Sample. PLOS ONE, 12(1): e0169839. $\underline{\text { doi:10.1371/journal.pone.0169839 }}$

Bennett, N. y O'Donohue, W. (2014). The Construct of Grooming in Child Sexual Abuse: Conceptual and Measurement Issues. Journal of Child Sexual Abuse, 8, 957-976. doi:10.1080/10538712.2014.960632

Berger, J. \& Milkman, K. (2012). What Makes Online Content Viral? American Marketing Association. 49, 192-205.

Buran, O. \& Dogan, A. (2019). The Relationship between Social Media Addiction and Self-Esteem among Turkish University Students. Addicta: the Turkish Journal on Addictions, 6(1), 175-190.

Cantor, M., Pérez, E., \& Carrillo, S. (2018). Redes sociales e identidad social. Aibi Revista de Investigación, Administración e Ingeniería, 6(2), 73-81.

Carbonell, X. \& Oberst, U. (2015). Las redes sociales en línea no son adictivas. Aloma, 33(2), 13-19.

Carbonell, X., Chamarro, A., Oberst, U., Rodrigo, B., \& Prades, M. (2018). Problematic Use of the Internet and Smartphones in University
Students: 2006-2017. International Journal Environmental Research and Public Health, 15(3), 475.

Carr, N. (2013). Superficiales. Madrid: Taurus.

Carrotte, E.R., Vella, A.M., \& Lim, M.S. (2015). Predictors of "Liking" Three Types of Health and Fitness-Related Content on Social Media: A Cross-Sectional Study. Journal of Medical Internet Research, 17(8), e205. doi:10.2196/jmir.4803

Cookingham, M. \& Ryan, G. (2015). The Impact of Social Media on the Sexual and Social Wellness of Adolescents. Journal of Pediatric and Adolescent Gynecology, 28(1), 2-5.

Challco, K., Rodríguez, S, \& Jaimes, J. (2016). Riesgo de adicción a redes sociales, autoestima y autocontrol en estudiantes de secundaria. Revista Científica de Ciencias de la Salud, 9(1), 9-15.

Chou, H. \& Chou, C. (2019). A quantitative analysis of factors related to Taiwan teenagers' Smartphone addiction tendency using a random sample of parent-child dyads. Computers in Human Behavior, 99, 335-344. doi:10.1016/j.chb.2019.05.032

De la Villa, M. \& Suarez, C. (2016). Factores de riesgo en el uso problemático de Internet y del teléfono móvil en adolescentes españoles. Revista Iberoamericana de Psicología y Salud, 7, 69-78.

Dias, P., Goncalves, S, García del Castillo-López, A., García-Castillo, F., García del Castillo, J.A., López-Sánchez, C., Ramos, I., \& Quiles, M.C. (2019). Móviles y redes sociales: un estudio con jóvenes. Book of Abstrac 5th International Congress of Clinical and Health Psychology on Children and Adolecents, 83. Madrid: Pirámide.

Diaz, V. (2011). Mitos y realidades de las redes sociales. Prisma Social 6, 1 26.

Estévez, A., Urbiola, I., Iruarriazaga, I., Onaindia J., \& Jauregui, P. (2017) Emotional Dependency in Dating Relationships and Psychological Consequences of Internet and Mobile Abuse. Anales de Psicología, $33(2), 260-268$

Ferreiro, E. (2015). La transformación del tiempo en la sociedad del conocimiento: una (des) aproximación teórica. Revista Contribuciones a las Ciencias Sociales, 29. Recuperado de http://www.eumed.net/rev/cccss/2015/03/tiempo.htm

Frost, R.L. \& Rickwood, D.J. (2017). A systematic review of the mental health outcomes associated with Facebook use. Computers in Human Behavior, 76, 576-600. doi:10.1016/j.chb.2017.08.001

García del Castillo, J.A. (2013). Adicciones tecnológicas: el auge de las redes sociales. Revista Health and Addictions/Salud y Drogas, 13(1), 5-14.

García del Castillo, J.A. (2015). Concepto de vulnerabilidad psicosocial en el ámbito de la salud y las adicciones. Revista Health and Addictions/Salud y Drogas, 15(1), 5-14.

Gardner, H. \& Davis, K. (2014). La generación App. Barcelona: Paidós.

Golpe, S., Gómez, P., Braña, T., Varela, J., \& Rial, A. (2017). Relación entre el consumo de alcohol y otras drogas y el uso problemático de Internet en adolescentes. Adicciones, 29(4), 268-277. 
González, E. \& Martínez, N. (2018). Redes sociales como factor incidente en el área social, personal y académica de alumnos de Educación Secundaria Obligatoria. Tendencias Pedagógicas, 32, 133-146. doi:10.15366/tp2018.32.010

Gros, B. (2005). Adolescentes y videojuegos: el juego desde el jugador. Comunicación y Pedagogía, 208, 75-77.

Hawk, S., van den Eijnden, R., van Lissa, C., \& ter Bogt, T. (2019). Narcissistic adolescents' attention-seeking following social rejection: Links with social media disclosure, problematic social media use, and smartphone stress. Computers in Human Behavior, 92, 65-75.

Holland, G. \& Tiggemann, M. (2016). A systematic review of the impact of the use of social networking sites on body image and disordered eating outcomes. Body Image, 17, 100-110.

Karinthy, F. (1929). Chains. En línea: https://djir-courses.wdfiles.com/local-files/soc180\%3Akarinthy-chain-links/Karinthy-ChainLinks 1929.pdf

Khan, S., Gagné, M., Yang, L., \& Shapka, J. (2016). Exploring the relationship between adolescents' self-concept and their offline and online social worlds. Computers in Human Behavior, 55(B), 940-945.

Kramer, A.D.I., Guillory, J.E., \& Hancock, J.T. (2014) Experimental evidence of massive-scale emotional contagion through social networks. Proceedings of the National Academy of Sciences, 111(24), 8788-8790.

Li, M. \& Lu, L. (2017). La influencia de la adicción al teléfono móvil en la calidad de sueño de estudiantes secundarios dejados atrás: el rol mediador de la soledad. Revista Argentina de Clínica Psicológica, XXVI(1), 71-81.

Li, P., Chang, L., Chua, T., \& Loh, R. (2018). "Likes" as KPI: An examination of teenage girls' perspective on peer feedback on Instagram and its influence on coping response. Telematics and Informatics, 35(7), 1994-2005.

Li, X., Kawachi, I., Buxton, O., Haneuse, S., \& Onnela, J. (2019). Social network analysis of group position, popularity, and sleep behaviorsamong U.S. adolescents. Social Science \& Medicine, 232, 417-426.

Livingstone S. \& Smith P.K. (2014). Annual research review: Harms experienced by child users of online and mobile technologies: the nature, prevalence and management of sexual and aggressive risks in the digital age. Journal of Child Psychology Psychiatry, 55(6), 635654.

Lizárraga, J., Rodelo, J., Tovar, J., \& Aguiano, C. (2015). Las redes sociales y su influencia en el rendimiento escolar de los estudiantes de nivel superior. RITI Journal, 3(5), 14-18.

López-Fernández, O., Honrubia-Serrano, L., Freixa-Blanxart, M., \& Gibson, W. (2014). Prevalence of problematic mobile phone use in British adolescents. Cyberpsychology Behavior and Social Networking, 17(2), 91-98. doi:10.1089/cyber.2012.0260

López-Sánchez, C. \& García del Castillo, J. A. (2017). La familia como mediadora ante la brecha digital: repercusión en la autoridad. Revista Colombiana de Ciencias Sociales, 8(1), 101-117. doi:10.21501/22161201.1928
Lucio, L. (2012). Conductas de acoso en Facebook en estudiantes de preparatoria y facultad. De la adicción a la transmisión de emociones negativas en la red social. Diálogos de Educación, 3(4), 1 16.

Malo, S., Martín M.M., \& Viñas, F. (2018). Uso excesivo de redes sociales: Perfil psicosocial de adolescentes españoles. Comunicar, 26(56). doi:10.3916/C56-2018-10

Marino, C., Vieno, A., Pastore, M., Albery, I.P., Frings, D., \& Spada, M.M. (2016). Modeling the contribution of personality, social identity and social norms to problematic Facebook use in adolescents. Addictive Behaviors, 63, 51-56. doi:10.1016/j.addbeh.2016.07.001

Martinez, J., Facundo, F., Cardenas F., Castillo, M., \& Garcia, K. (2017). Influencias urbanas y la exposición de drogas en facebook con el consumo de drogas. Journal Health NPEPS, 2(1), 161-175.

McCann, M., Broccatelli, C., Moore, L., \& Mitchell, K. (2018). Distribution of sexual health knowledge and attitudes in adolescent social networks: social network analysis of data from the STIs and Sexual Health feasibility study. The Lancet, 392(2), s60.

Milgram, S. (1967). The Small-World Problem. Psychology Today, 1(1), 6067.

Mingoia J., Hutchinson, A.D., Wilson, C., \& Gleaves, D.H. (2017). The Relationship between Social Networking Site Use and the Internalization of a Thin Ideal in Females: A Meta-Analytic Review. Frontiers in Psychology, 8, 1351. doi:10.3389/fpsyg.2017.01351

Moreno, N., González, A., Torres, A., \& Araya, J. (2017). Alfabetización digital a padres de familia en el uso de las redes sociales. Alteridad, 12(1), 8-19.

Navarro, E., De Anda, P., Gámez, M., Candía, J., \& Guzmán, F. (2016). Exposición a contenidos de uso de alcohol en Facebook y consumo de alcohol en adolescentes. Revista Enfermería Herediana, 9(1), 4349.

Nesi, J., Rothenberg, W., Hussong, A., \& Jackson, K. (2017). Friends' AlcoholRelated Social Networking Site Activity Predicts Escalations in Adolescent Drinking: Mediation by Peer Norms. Journal of Adolescent Health, 60(6), 641-647.

Park, M., Sun, Y., \& McLaughlin, M.L. (2017). Social Media Propagation of Content Promoting Risky Health Behavior. Cyberpsychology. Behavior and Social Networking, 20(5), 278-85. doi:10.1089/cyber.2016.0698

Pedrero, E., Ruiz, J.M., Rojo, G., Llanero, M., Pedrero, J., Morales S., \& Puerta, C. (2018). Tecnologías de la Información y la Comunicación (TIC): uso problemático de Internet, videojuegos, teléfonos móviles, mensajería instantánea y redes sociales mediante el MULTICAGETIC. Adicciones, 30(1), 19-32.

Pérez-Torres, V., Pastor-Ruiz, Y., \& Abarrou-Ben-Boubaker, S. (2018). Los youtubers y la construcción de la identidad adolescente. Comunicar, 26(55), 61-70.

Peris, M., Maganto, C., \& Kortabarria, L. (2013). Autoestima corporal, publicaciones virtuales en las redes sociales y sexualidad en 
adolescentes. European Journal of Investigation in Health, Psychology and Education, 3(2), 171-180.

Pertegal, M.A., Oliva, A., \& Rodríguez, A. (2019). Revisión sistemática del panorama de investigación sobre redes sociales: taxonomía sobre experiencia de uso. Comunicar, 26(60). doi:10.3916/C60-2019-08

Puerto, M., Rivero, D., Sansores, L., Gamboa, L., \& Sarabia, L. (2015). Somnolencia, hábitos de sueño y uso de redes sociales en estudiantes universitarios. Enseñanza e Investigación en Psicología, 20(2), 189-195.

Radovic, A., Gmelin, T., Stein, B., \& Miller, E. (2017). Depressed adolescents' positive and negative use of social media. Journal of Adolescence, 55, 5-15.

Raudsepp, L. \& Kais, K. (2019). Longitudinal associations between problematic social media use and depressive symptoms in adolescent girls. Preventive Medicine Reports, 15, Article 100925.

Restrepo, J.E. \& Castañeda, T. (2018). Riesgo de trastorno de la conducta alimentaria y uso de redes sociales en usuarias de gimnasios de la ciudad de Medellín, Colombia. Revista Colombiana de Psiquiatría. doi:10.1016/j.rcp.2018.08.003.

Rissoan, R. (2016). Redes sociales. Comprender y dominar estas nuevas herramientas de comunicación. Barcelona: Ediciones ENI.

Rojas Jara, C., Ramos Vera, J., Pardo-González, E., \& Henríquez-Caroca, F. (2018). Adicción a internet en adolescentes: una breve revisión. Drugs and Addictive Behavior, 3(2), 267-281. doi:10.21501/24631779.2876

Salas, E. \& Escurra, M. (2014). Uso de redes sociales entre estudiantes universitarios limeños. Revista Peruana de Psicología y Trabajo Social, 3(1), 75-90.

Sampasa, H. \& Chaput, J.P. (2016). Use of social networking sites and alcohol consumption among adolescents. Public Health 139, 88-95.

Sampasa, H. \& Hamilton, H.A. (2015). Social networking sites and mental health problems in adolescents: The mediating role of cyberbullying victimization. European Psychiatry, 30(8), 1021-1027.

Sampasa, H. \& Hamilton, H.A. (2018). Use of social networking sites, electronic cigarettes, and waterpipes among adolescents. Public Health, 164, 99-106.

Santos, R. (2019). Interculturalidad, identidad personal y redes sociales: caminos de atestación de sí. Ciencia y Sociedad, 43(2), 25-33.

Sanz Arazuri, E., Alonso Suiz, R.A., Sáenz de Jubera Ocón, M., Ponce de León Elizondo, A., \& Valdemoros San Emeterio, M. A. (2018). Ocio, redes sociales y estudiantes españoles. Educación XX1, 21(2), 59-78, doi:10.5944/educXX1.19538

Seo, D. \& Ray, S. (2019). Habit and addiction in the use of social networking sites: their nature, antecedents, and consequences. Computers in Human Behavior, 99, 109-125. doi:10.1016/j.chb.2019.05.018

Serrano Puche, J. (2013). Vidas conectadas: tecnología digital, interacción social e identidad. Historia y Comunicación Social, 18, 353-364.
Singleton, A., Abeles, P., \& Smith, I.C. (2016). Online social networking and psychological experiences: The perceptions of young people with mental health difficulties. Computers in Human Behavior, 61, 394 403.

Soh, P., Chew, K., Koay, K., \& Ang, P. (2018). Parents vs peers' influence on teenagers' Internet addiction and risky online activities. Telematics and Informatics, 35, 225-236.

Tang, J.H., Chen, M.C., Yang, C.Y., Chung, T.Y., \& Lee, Y.A. (2016). Personality traits, interpersonal relationships, online social support, and Facebook addiction. Telematics and Informatics, 33, 102-108. doi:10.1016/j.tele.2015.06.003

Tang, C. \& Koh, Y. (2017). Online social networking addiction among college students in Singapore: Comorbidity with behavioral addiction and affective disorder. Asian Journal of Psychiatry, 25, 175 178.

Throuvala, M., Griffiths, M.D., Rennoldson, M., \& Kuss, D.J. (2019). Motivational processes and dysfunctional mechanisms of social media use among adolescents: A qualitative focus group study. Computers in Human Behavior, 93, 164-175.

Turner, P.G. \& Lefevre, C.E. (2017). Instagram use is linked to increased symptoms of orthorexia nervosa. Eat Weight Disord, 22(2), 277-284. doi:10.1007/s40519-017-0364-2

Watts, D. (2003). Six degrees. The science of a connected age. London: William Heinemann. 